\title{
Reflections on case studies, modelling and theory building
}

\author{
M. Moglia ${ }^{a}$, K. Alexander ${ }^{\mathrm{b}}$, P. Perez \\ Affiliations: ${ }^{a}$ Urban and Industrial Water Research Program, CSIRO Land and Water, Victoria, ${ }^{b}$ CSIRO \\ Ecosystem Sciences, Canberra, ACT, Australia ${ }^{c}$ SMART Infrastructure Facility, University of Wollongong, \\ New South Wales,
}

\section{Email: magnus.moglia@.csiro.au}

This paper provides a discussion on how case study research fits into the bigger picture of theory building in the social sciences. In particular, it discusses how case study research, as opposed to more classical quantitative (often statistical) approaches contribute to the progression of collective learning in science. As such, for arguments sake, in this paper these approaches (i.e. qualitative vs. quantitative) are put into a competitive relationship. It is acknowledged that such competitive relationship does not exist in theory, as they are often considered to be complementary, but in practice qualitative research is often more difficult to publish; and tends to be more difficult to defend. Therefore the authors believe there is a need to better recognise the role of case study research in the scientific process; and in light of recent development in participatory modelling to collectively re-evaluate how case study research contributes to the progression of scientific learning?

The deductive statistical approach in social science, as it was defined in a classic exchange of articles and ideas by mathematician Bernoulli and Galton in the $18^{\text {th }}$ century is to ascertain a posteriori through knowledge based on experience, in situations when we cannot determine a "priori" (independent of experience) by statistical analysis of a number of similar instances. This assumes events in the future will follow a similar pattern as in the past, and relies on several important assumptions which may inherently limit applicability. Firstly, the output of "priori" calculations is invariably correlations of relationship similarities rather than identified as causative factors. Secondly, the assumption of "similar conditions" is problematic because by definition this requires causal surmise and cannot be ascertained purely through statistical calculations. Consequently, social science research based on statistics alone is incomplete and requires causal explanations, suggesting the need for theoretical formulations. Whilst there are modern statistical techniques to alleviate some of these issues, such as capture-recapture, jack-knife and bootstrap; this shows that social science based on statistics alone is incomplete but requires causal explanations which in turn require the formulation of theory.

Conversely, inductive case study research has been criticized largely for being unable to result in laudable generalisations. The authors suggest case studies are suitable for developing human understanding of issues, and producing context dependent knowledge necessary for the formulation of theory. Specifically, case study research can be used for abductive reasoning whereby hypothetical explanations, A, of observations are found that are sufficient but do not necessarily explain observations B. Traditional scientific method would dictate that such a theory is tested using empirical falsification, but this is not always possible due to difficulties of observation. It is clear that the research process requires both (inductive) case study research as well as (deductive) statistical approaches. Furthermore, there is a need for a trained mind to synthesize the results and develop and choose the "best" theory / model to explain observations (abductive reasoning).

Participatory modelling with its mix of qualitative and quantitative methods places it within two major paradigms: generalisation via theory and statistics vs. context specific knowledge via case study. The question is whether participatory modelling can be the bridge between two main paradigms, by allowing for the development of general theories through the immersion of oneself in the specific. Limitations to possible applications remain, as participatory modelling is usually warranted and feasible only in certain cases, and is ethically dependant on circumstance. The authors argue that participatory modelling has not been developed with these purposes in mind, but serves merely as a thought provoking exemplar that is hopefully paving the way for the next generation of research methods. If this is to be the case, participatory modelling requires a strong theoretical foundation considering its place in epistemology and scientific method.

Keywords: Case study research, participatory modelling, social science 


\section{STATISTICS BASED SOCIAL SCIENCE}

This paper provides the authors' opinions and musings on statistics, case study research and how the scientific role of case study research may change as a consequence of the growing maturity of participatory modelling. As such, the tone is informal, and sometimes a bit provocative, and hopefully the reader will accept this. First some muses about statistics in social science. For argument's sake, a somewhat provocative stance is presented by deconstructing a naive representation of statistics based on an example within social sciences. This serves the purpose of showing the need for case study research as a complement and critical tool. Statistics as a critical tool has been used in most fields of social science, particularly in economics and psychology, but also in fields such as political science, sociology and anthropology. Statistically based social science has assumptions that it is possible to observe multiple instances of essentially the same (or similar) event, and then to make inferences on frequencies of particular behaviours, actions or events, as well correlations between these, often assuming that correlations represent causal relationships.

\subsection{Inadequacy of correlations}

For example, in the dawn of these types of analyses, statisticians were interested in the ages when individuals died; mainly for life insurance purposes; and here we describe an example drawn from Livio (2009). Large "life tables" were set up with the number of people in a population at different ages. By doing this, attempts were made to ascertain aspects of what is now known as reliability theory (Crowder et al., 1991) including; survival rates, hazard rates, probability density functions and cumulative distribution functions. These calculations were based on the possibility of estimating the probability of death at a given age. Similar analysis is now used for analysing stocks of animals, as well as for infrastructure such as bridges, car components and pipes. The problem with this form of analysis is that "death rates" are dependent on a range of factors outside of the scope of the analysis, varying considerably over time and from location to location. Consequently, the models that were developed were unable to offer a causal explanation of the reasons for "death". Following the explorations of "death rates", statisticians were interested in the "average man", plotting quantifiable attributes such as height, weight, length of the forearm etc, giving rise to the concept of correlation, introduced by Sir Francis Galton (1889):

The length of the forearm is correlated with the stature, because a long cubit usually implies a tall man. If the correlation between them is very close, a very long forearm would usually imply a very tall stature, but if it were not very close, a very long forearm would be on the average associated with only a tall stature and not a very tall one; while, if it were nil, a very long forearm would be associated with especial stature, and therefore, on the average, with mediocrity.

As a simplistic concept, this has some predictive power. While Karl Pearson formulated this in mathematical terms, correlations could exist for a range of reasons that do not necessarily indicate causality between the explored factors, even though causality is one plausible explanation (Stigler, 1989). It is noted that for the case of two or three inter-related variables, Granger (1969) has provided statistical tools to evaluate causality. Furthermore, this may seem like an observation that is well known to most of those that are familiar with statistics; but it is the authors' experience that misunderstandings start appearing in the area of hypothesis testing where tests are based on concepts similar to correlation, and all that is ever achieved is simply rejection, or no rejection of a null hypothesis. A hypothesis is never proven to be correct, but it may only be possible to reject it. Many hypotheses are likely to be consistent with data, but it requires a trained mind to find the most likely theory that explains data. Abductive reasoning is by necessity a critical part of the scientific process.

\subsection{Concern over observations}

Statistically based social science has improved since the $18^{\text {th }}$ Century, and there are several tools using statistical approaches for confirmatory modelling (confirm and quantify hypothesised causal relationships) as well as exploratory modelling (identify plausible hypothesis that may explain data). In Experimental Economics, controlled experiments in the field or in a laboratory are usually devised for experiments to confirm and/or quantify a particular hypothesis. In structural equation modelling, a large data set is typically analysed to confirm and quantify causal relationships, and can also be used to further theory development. Whilst structural equation modelling is a powerful method of enquiry, the disadvantage with large data requirements is that we can only consider in our causal models that which we can observe.

Personal experiences and behavioural responses can be causally modelled. For example, external environmental stimuli trigger emotional responses, which may also be impacted by extenuating the initial emotional response 
onto another external stimuli Exploration of these linked experiences and introspection to appraise the experience requires considerable discipline and self-awareness that may be close to impossible to achieve. It is often difficult to get enough observations to provide sufficient scope for use in statistics based social science; especially if one does not rely on case study research and more anthropological style of research. The complexity of human values, beliefs, behaviours and responses gives rise to difficulties in assessing social mores and hence humans could be considered as fickle, uninformed, inconsistent and unaware of self, and hence the reliability of survey data may be questioned. In short, whilst it is literally impossible to ask atoms why they behave in certain ways, it is possible to ask humans this question; but deciphering their answers as macro level outputs of a complex and adaptive inner world is often a difficult and time consuming task.

\subsection{Limitations of statistics in social science}

As argued, the use of statistics in social science is limited by several factors; the inadequacy of correlation; the difficulty of recreating situations; and concerns about the accuracy and power of personal observations. Limitations arise from the inability to simplify rich descriptions of situations and problems that are in reality far more complex, dynamic and messy and not suitable for conversion. For example, it seems ludicrous to describe in statistical terms the classic anthropological works on voodoo by McCarthy-Brown (2001) which has a wealth of richness of description and information. Whilst there may be aspects of this work that can be described using statistics such as for arguments sake correlating moods and actions of voodoo priestess Mama Lola, using content analysis, classification and statistical tests, however such descriptions will remain incomplete. The point is that statistical and deductive approaches are inadequate by themselves for the development of social science; and need to be complemented with other approaches. Methods for collecting data for complex social science models (such as Agent Based Models) may include expert knowledge, surveys, interviews, and participant observation (Smajgl et al., 2011). Case study research is one such approach, which has strengths where statistics has limitations and weaknesses where statistics has strengths.

\section{CASE STUDY RESEARCH}

Case study research is a common method in social science which in most ways is very different to the statistical approach in that it tends to involve an in-depth and situational examination of a single case. Qualitative data is used extensively reducing the limitations on observational capacity alone, however some argue there is too much scope for the researcher's own interpretations. Case study research is often subject to criticism because of the misconception that one cannot generalise from a single case, which by some is seen as the main purpose of social science (Flyvbjerg, 2006). Flyvbjerg in support of case study research dismisses five major misunderstandings of attributes of case study research and in their place statements of fact as listed (Flyvbjerg 2006: 221-241):

Misunderstanding 1: General, theoretical (context-independent) knowledge is more valuable than concrete, practical (context-dependent) knowledge;

Statement 1: Predictive theories and universals cannot be found in the study of human affairs. Concrete, context-dependent knowledge is, therefore, more valuable than the vain search for predictive theories and universals;

Misunderstanding 2: One cannot generalise on the basis of an individual case; therefore, the case study cannot contribute to scientific development;

Statement 2: One can often generalise on the basis of a single case, and the case study may be central to scientific development via generalisation as supplement or alternative to other methods. But formal generalisation is overvalued as a source of scientific development, whereas 'the force of example' is underestimated;

Misunderstanding 3: The case study is most useful for generating hypotheses; that is, in the first stage of a total research process, whereas other methods are more suitable for hypotheses testing and theory building;

Statement 3: The case study is useful for both generating and testing of hypotheses but is not limited to these research activities alone; 
Misunderstanding 4: The case study contains a bias toward verification, that is, a tendency to confirm the researcher's preconceived notions;

Statement 4: The case study contains no greater bias toward verification of the researcher's preconceived notions than other methods of inquiry. On the contrary, experience indicates that the case study contains a greater bias toward falsification of preconceived notions than toward verification;

Misunderstanding 5: It is often difficult to summarise and develop general propositions and theories on the basis of specific case studies.

Statement 5: It is correct that summarising case studies is often difficult, especially as concerns case process. It is less correct as regards case outcomes. The problems in summarising case studies, however, are due more often to the properties of the reality studied than to the case study as a research method. Often it is not desirable to summarise and generalise case studies. Good studies should be read as narratives in their entirety.

If one accepts these statements, case study research has been shown to be a powerful tool of the social scientists' capability, and overcoming some shortcomings of the statistical methods, i.e. better observational capacity and less simplistic representations of causality; and increased potential for abductive reasoning. Case study research provides ample opportunity to develop a good understanding of complex problems; which provides a good basis for abductive reasoning whereby the theory that appears to be most likely to explain a given situation / phenomenon is chosen as the preferred theory. However as shown in the common misunderstandings that are described above, it is commonly (and wrongly) believed that case study research is unreliable and of little value.

\section{DEVELOPING AND TESTING THEORY}

Theories can account for causal explanations which in turn (usually in combination with mathematics) allow for generalisation to a larger population. For example, it was Newton's musings over a few limited observations that allowed him to formulate his theory of gravity in mathematical terms. The theoretical formulation of his theory in mathematical terms in turn allowed for prediction and generalisation far beyond the domains of the initial observations. Similarly, successful theory developers in management theory and psychology report how their research is inductive and firmly based on an initial step of "immersing" oneself in a substantial body of observations and data - often involving a deep understanding of individual instances using case study research. Immersion requires creative synthesis, inductive reasoning (because there is typically incomplete data) as well as hypothesis development and critical evaluation by peers. Other aspects may include thought experiments, consistency checks and empiricism. Statistics remains an important tool in this process, but in itself provides insufficient explanations.

At the centre of scientific endeavours are the tasks of developing and testing theory as a process for collective learning. Usually, theory building is the development of causal relationships (i.e. cause and effect). Such statements of cause and effect are based on experience, but the problem is that there are many types of cause and effect relationships that may explain the same observations. For example, one may deduce that morning has arrived from observations of the sun rising on the horizon. This observation has good predictive capacity, but is essentially useless as the explanation is more complex. The fundamental physics explaining morning sun rise was found by scientists over a long time frame to be associated with the planets orbiting the sun, and the rotation of the earth, bound by gravitational forces. Consequently, the question becomes: what is a good theory?

A good theory tends to allow for causal explanations which in turn allow for predictions, which if combined with reliable observations can be used for validation via falsification as argued by often criticised logical positivist Popper (1963). Falsification is often achieved using statistical methods, but may also be achieved via case study (one instance or reliable observation may be used as an example for the purposes of falsification). Theories are also tested for internal consistency; i.e. lack of errors and conservation of things like correlations. Many also argue that a theory (or model) is good if it is useful, but its usefulness often remains unknown for some time as impacts can take a long time to mature and to become apparent. Furthermore, validation and verification of theory is typically tested through a peer-review process, usually of academic peers, and sometimes, in particular in participatory modelling (see late for more discussion about this), also by stakeholders in a study context. 
But how does one develop good theory? It seems even the theories of gravitation and basic physics have tended to be developed by individuals with particular skills of inductive reasoning, i.e. by proceeding from the particular to the general. Good theory developers often have to find suitable causative explanations based on particular and limited observations, in order to develop theories that allow for general predictions. The same seems to be true in social science where Locke (2007) promotes a particular research process based on induction, i.e. Inductive Theory Building, on the basis that it has provided the more successful theories and models in management theory and on human interactions. This opinion is reinforced by agreement as described in a collection of essays on research experiences by successful theory builders (Smith and Hitt, 2005), such as developers of Social Cognitive Theory (Bandura, 1977), the Theory of Double Loop Learning (Argyris and Schön, 1996), and Goal Setting Theory (Locke and Latham, 2002). Their experiences and conclusions about the research process has been synthesised by Locke (2007).

What can be learnt about theory building is that science does not progress by suddenly espousing theory, or a model, from total ignorance to omniscience. Instead knowledge and science develop incrementally, and errors are made and corrected. In this process, counterfactuals, i.e. alternative realities, are imagined and explored in a process of abductive reasoning. A classic example of abductive reasoning, deduces why a lawn is wet. Several reasons have explanatory power, either it recently rained last night, or perhaps the sprinklers have been left on. Abductive reasoning is probabilistic in that judgments and decisions are made about the most likely explanation. In doing this, our knowledge of conditions may be drawn upon, for example knowledge of weather forecasts, or witness of storms etc. By abductive reasoning, conclusions can be drawn that the likelihood of rain was the causative agent involved in wetting the lawn. This provides an example of the need for an informed guessing strategy in finding the most likely explanation of observations - where individual skill and knowledge is critical. In light of this, it is not surprising that two critical steps in the process of Inductive Theory Building is firmly based on developing a substantial body of observations and data, and to make theory building a careful, painstaking, and gradual process (Locke, 2007, p.884):

Anyone can invent a theory off the cuff. But for it to be tied firmly to reality and therefore have lasting value, a valid theory needs to be gradually built from an accumulating body of evidence. There may be exceptions if a relevant and substantial body of knowledge already exists and a genius like Einstein is able to integrate it.

The steps of Inductive Theory Building suggested by (Locke, 2007) are to:

1. Start with a statement of valid philosophical axioms that are seen as primary self-evident facts of reality, and which cannot be further deconstructed;

2. Develop a substantial body of observations and data, and this is not necessarily quantitative data;

3. Formulate valid concepts and definitions on the basis of similarity and careful consideration of observations;

4. Look for evidence of causality and identify plausible causal mechanisms (as described above);

5. Integrate the totality of findings and concepts into a non-contradictory whole (i.e. make theory internally consistent);

6. Identify the domain and boundary conditions for the theory; and

7. Make theory building a careful, painstaking and gradual process. This is an almost algorithmic kind of process which centres on the principles of firstly maximising the capacity for abductive reasoning, and secondly to maximise the capacity for theories to withstand testing.

With this in mind, it appears that case study research can be useful for a number of the steps in the theory building process and also does not typically presuppose concepts or causal mechanisms. Case study research will help in formulating valid concepts, and identify plausible counterfactuals, which can form the basis of abductive reasoning. This is aligned with Grounded Theory according to which, adapted from Glaser and Strauss (1967): 1) Everything is considered to be data which can be used by the researcher to generate concepts, including emails, lectures, conversations, books etc, etc. 2) All data is coded to extract concepts, which are classified and this is an iterative and ongoing process as the theory sharpens and new concepts emerge. 3) All concepts are narrowed down by selecting the core variable, and this is further refined as learning improves. 
Using the same line of reasoning, it can be argued that a danger of practicing statistically based social science is the avoidance of the messy realities that we are trying to understand with incomplete information (inductive reasoning). If we are not careful, our biases and prejudices are more likely to guide the formulation and selection of plausible counterfactuals (abductive reasoning). At the same time, it is acknowledged that statistics and probabilistic (deductive) reasoning can be powerful tools for making sense of, and finding patterns in complex data.

\section{HOW WILL PARTICIPATORY MODELLING CHANGE CASE STUDY RESEARCH?}

Participatory modelling is a relatively new field, which is gaining increasing momentum and maturity; but is perhaps not consistently defined within its discipline (Voinov and Bousquet, 2010). It applies post-modern ideas, in a kind of democratisation of the research process, in that it allows stakeholders to take part in the modelling process (Dray et al., 2006, Voinov and Bousquet, 2010). Whilst the ideas vary within participatory modelling, there are some key commonalities, i.e. 1) heavily relying on case study research, 2) utilising stakeholders for peer-review and contributing in the model building process (i.e. assessing "similarity to reality" of alternative counterfactuals), and 3) primarily assessing models/theories on the basis of their usefulness rather than their validity (Jones et al., 2009). It is clear that this approach is useful in a local context and has proven to be useful in other situations, as is for example discussed by Daniell (2008) or Jones et al (2009) showing for example that it helps legitimise identified strategies, allows for co-engineering of solutions, supports creating a common frame of reference, and provides a more grounded decision analysis process. Furthermore, in this way, the authors believe that it is likely that stakeholders collectively have greater capability for abductive reasoning than do individual researchers, or groups of researchers.

In light of this, the authors posit the questions: "Is it possible that the participatory Modelling approach can change the role and process of case study research, and hence providing a way to successfully blend qualitative and quantitative research?" And if so, what is the method for participatory modelling that is most effective for "good" model building? And, to move further, how does the protocol for undertaking and reporting on case study research (in a participatory modelling framework) need to change? The reliance on case study research is critical, but to allow for generalisation, there needs to be robust methods for parameterisation (Smajgl et al., 2011) and may sometimes need complementary experiments to improve empirical calibration and for validation (Heckbert et al., 2010, Janssen and Ostrom, 2006); as well as evaluation protocols (Jones et al. 2009) and providing the facilities for open and fair external evaluation and testing (Korfmacher, 2001).

Probably more than anything, it seems that case study research is due to its complexity and contextual nature, difficult to review; and this may be the reason why it is sometimes criticized (if one can't trust it, how can it be generalised?). Participatory modelling suggests that stakeholders take a role in the review process, which is a good step forward in the democratisation of this process. However, it can be argued that there needs to be further transparency, openness and rapid review, and perhaps practitioners of case study research should find new ways of facilitating this collective learning. Much could be learnt from the IT community where numerous (moderated) mechanisms (blogs, Slashdot, etc) exist for rapid, open and transparent review and discussion within a specialised community. If one can trust case study research, it is a powerful tool; not least for its capacity to help abductive reasoning. Better abductive reasoning is likely to fast-track scientific learning by consistently choosing more accurate theories and models of reality.

\section{REFERENCES}

Argyris, C. \& Schön, D. (1996) Organisational Learning II: Theory, Learning and Practice, Reading Ma, U.S., Addison-Wesley Publishing.

Bandura, A. (1977) Social learning theory, Englewood Cliffs, New Jersey, USA, Prentice-Hall.

Crowder, M.J., Kimber, A.C., Smith, R.L. \& Sweeting, T.J. (1991) Statistical analysis of reliability data, London, U.K., Chapman \& Hall.

Daniell, K.A. (2008) Co-engineering participatory modelling processes for water planning and management. $\mathrm{PhD}$, The Australian National University. 
Moglia et al., Reflections on case studies, modelling and theory building

Dray, A., Perez, P., Jones, N., Le Page, C., D'Aquino, P., White, I. \& Auatabu, T. (2006) The AtollGame Experience: from Knowledge Engineering to a Computer-Assisted Role Playing Game. Journal of Artificial Societies and Social Simulation, 9(1).

Flyvbjerg, B. (2006) Five Misunderstandings About Case-Study Research. Qualitative Inquiry, 12(2), 219-245.

Galton, F.R.S. (1889) Correlations and their measurement, chiefly from anthropometric data. Nature, 39(1889 January 3), 238.

Glaser, B. \& Strauss, A. (1967) The Discovery of Grounded Theory: Strategies for Qualitative Research,, New York, U.S., Aldine Transaction.

Granger, C.W.J. (1969) Investigating causal relations by econometric models and cross-spectral; methods. Econometrica, 37(424-438.

Heckbert, S., Baynes, T. \& Reeson, A. (2010) Agent-based modeling in ecological economics. Annals of the New York Academy of Sciences, 1185(Ecological Economics Reviews), 39-53.

Janssen, M.A. \& Ostrom, E. (2006) Empirically Based, Agent-based models. Ecology and Society, 11(2), Art. 37.

Jones, N.A., Perez, P., Measham, T.G., Kelly, G.J., d'Aquino, P., Daniell, K.A., Dray, A. \& Ferrand, N. (2009) Evaluating Participatory Modeling: Developing a Framework for Cross-Case Analysis. Environmental Management, 44(6), 1180-1195.

Korfmacher, K.S. (2001) The politics of participation in watershed modeling. Environmental Management, 27(2), 161-176.

Livio, M. (2009) Is God a Mathematician?, New York, U.S., Simon \& Schuster.

Locke, E.A. (2007) The Case for Inductive Theory Building. Journal of Management, 33(6), 867-890.

Locke, E.A. \& Latham, G.P. (2002) Building a practically useful theory of goal setting and task motivation. American Psychologist, 57(9), 705-717.

McCarthy Brown, K. (2001) Mama Lola: a Vodou priestess in Brooklyn, LA, U.S., University of California Press.

Popper, K. (1963) Conjectures and Refutations, London, U.K., Routledge and Kegan Paul.

Smajgl, A., Brown, D.G., Valbuena, D. \& Huigen, M.G.A. (2011) Empirical characterisation of agent behaviours in socio-ecological systems. Environmental Modelling \& Software, 26(7), 837-844.

Smith, K.G. \& Hitt, M.A. (2005) Great Minds in Management: The Process of Theory Development New York, US, Oxford University Press.

Stigler, S.M. (1989) Francis Galton's Account of the Invention of Correlation. Statistical Science, 4(2), 73-86.

Voinov, A. \& Bousquet, F. (2010) Modelling with stakeholders. Environmental Modelling and Software, 25(11), 1168-1281. 